\begin{tabular}{lll}
\hline Ruchi Yadav, MD & Debasis Sahoo, MD, FCCP & Ruffin Graham, MD \\
Departments of Diagnostic Radiology and & Department of Pulmonary Medicine, & Section Head, Department of Diagnostic \\
Nuclear Medicine, Imaging Institute, Cleveland & Respiratory Institute, Cleveland Clinic; & Radiology, Imaging Institute, Cleveland Clinic; \\
Clinic; Clinical Assistant Professor, Cleveland & Clinical Assistant Professor, Cleveland Clinic & Assistant Professor, Cleveland Clinic Lerner \\
Clinic Lerner College of Medicine of Case & Lerner College of Medicine of Case Western & College of Medicine of Case Western Reserve \\
Western Reserve University, Cleveland, OH & Reserve University, Cleveland, OH & University, Cleveland, OH
\end{tabular}

\title{
Thoracic imaging in COVID-19
}

\section{ABSTRACT}

The typical findings of COVID-19 on chest radiography and computed tomography (CT) include bilateral, multifocal parenchymal opacities (ground-glass opacities with or without consolidation, and "crazy paving"). In most cases, the opacities are predominantly in the peripheral and lower lung zones, and several have rounded morphology. However, these imaging findings are not pathognomonic for COVID-19 pneumonia and can be seen in other viral and bacterial infections, as well as with noninfectious causes such as drug toxicity and connective tissue disease. Most radiology professional organizations and societies recommend against routine screening $\mathrm{CT}$ to diagnose or exclude COVID-19.

\section{KEY POINTS}

Chest radiography is considered an appropriate initial imaging diagnostic test for most patients with lower respiratory tract infection, including those suspected of having COVID-19. However, it is neither sensitive nor specific.

CT features of COVID-19 pneumonia are not pathognomonic. Hence, when making the diagnosis, CT findings must be integrated with the clinical presentation, exposure history, prevalence of COVID-19 in the community, and personal risk factors.

A normal result on chest $\mathrm{CT}$ cannot exclude the diagnosis of COVID-19, especially early after the onset of symptoms.

Professional societies have issued guidelines on imaging in COVID-19, and the field continues to evolve.

doi:10.3949/ccjm.87a.ccc032
7 HE LUNGS are the most common site of in1 fection in COVID-19, and progression to respiratory failure is the most common cause of death. In this brief summary we describe the role of thoracic imaging in COVID-19.

\section{CHEST RADIOGRAPHY IN COVID-19}

Chest radiography is considered an appropriate initial imaging diagnostic test for most patients with lower respiratory tract infection, including those suspected of having COVID-19. The radiographic abnormalities in COVID-19 mirror those on computed tomography (CT), demonstrating bilateral, peripheral, and midlower-lung-zone-predominant consolidation (Figure 1). ${ }^{1}$ However, in patients who have a high pretest probability of COVID-19, atypical findings such as diffuse interstitial changes or unilateral focal consolidation (Figure 2) should not dissuade the radiologist from suspecting an infection, including COVID-19, as a possible diagnosis.

In patients with progressive disease, the density and extent of parenchymal changes typically increase over time (Figure 3 ). The severity of chest radiographic findings peaks 10 to 12 days after the onset of symptoms. ${ }^{1}$

Unfortunately, most bacterial pneumonias also present as consolidation, and it is difficult to distinguish them from viral infections on chest radiography. The subtleties of rounded morphology and "crazy paving" associated with COVID-19 can only be appreciated on $\mathrm{CT}$ and not on plain chest radiographs. Cavitation within an airspace consolidation likely suggests a superadded infection.

Moreover, chest radiography has a high false-negative rate, especially in the early stage of infection, and should not be used as a screening tool to rule out COVID-19. In fact, baseline radiography has a lower sensitivity $(69 \%)$ 

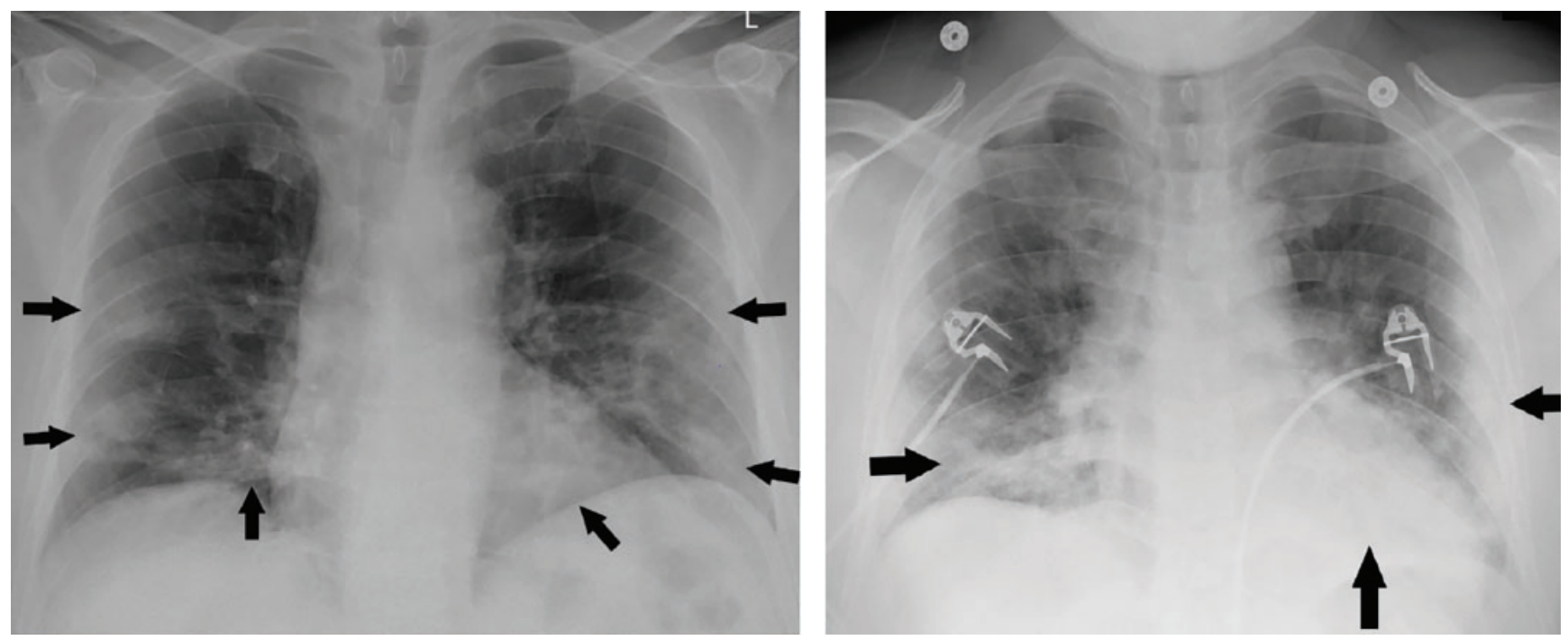

Figure 1. Portable chest radiographs of a patients with COVID-19 demonstrating classic bilateral, multifocal peripheral airspace opacities in the mid-lower-lung zones.
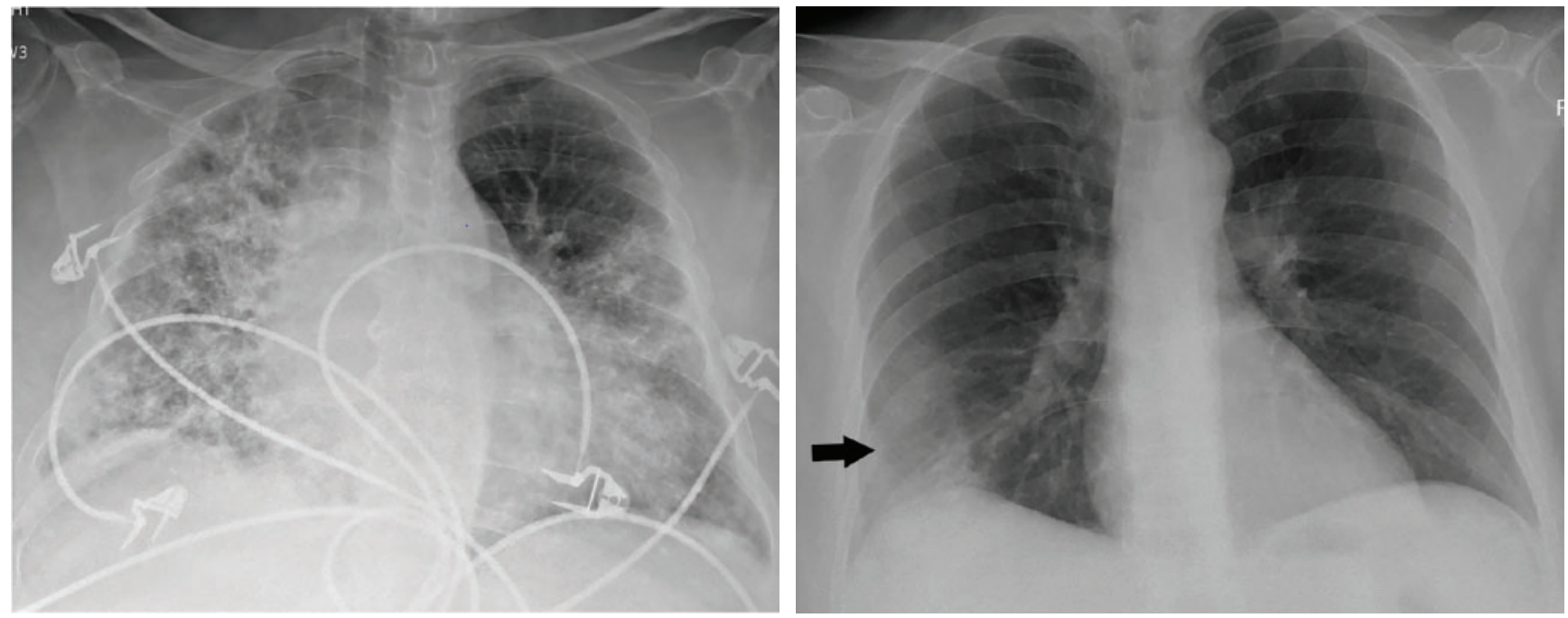

Figure 2. Portable chest radiographs of patients with COVID-19 demonstrating atypical features of diffuse bilateral interstitial changes $(A)$ and unilateral consolidation (B).

than initial reverse transcriptase polymerase chain reaction (RT-PCR) testing (91\%). ${ }^{1}$

\section{RADIOGRAPHY 'THROUGH GLASS' TO AVOID SPREADING THE VIRUS}

During the pandemic, our hospital (as well as many others in the United States) has employed a method of obtaining portable radiographs in cases of confirmed or suspected COVID-19 through the glass wall of the patient's room in the intensive care unit and in the emergency department.
With some minor technical modifications, the chest radiographs taken "through glass" are comparable to those obtained by the standard method (Figure 4). This technique has the potential to reduce the consumption of personal protective equipment by radiology technicians and to reduce the risk of machine contamination. ${ }^{2}$

\section{COMPUTED TOMOGRAPHY IN COVID-19}

Features of COVID-19 pneumonia on CT are not pathognomonic and are similar to those 


\section{YADAV AND COLLEAGUES}
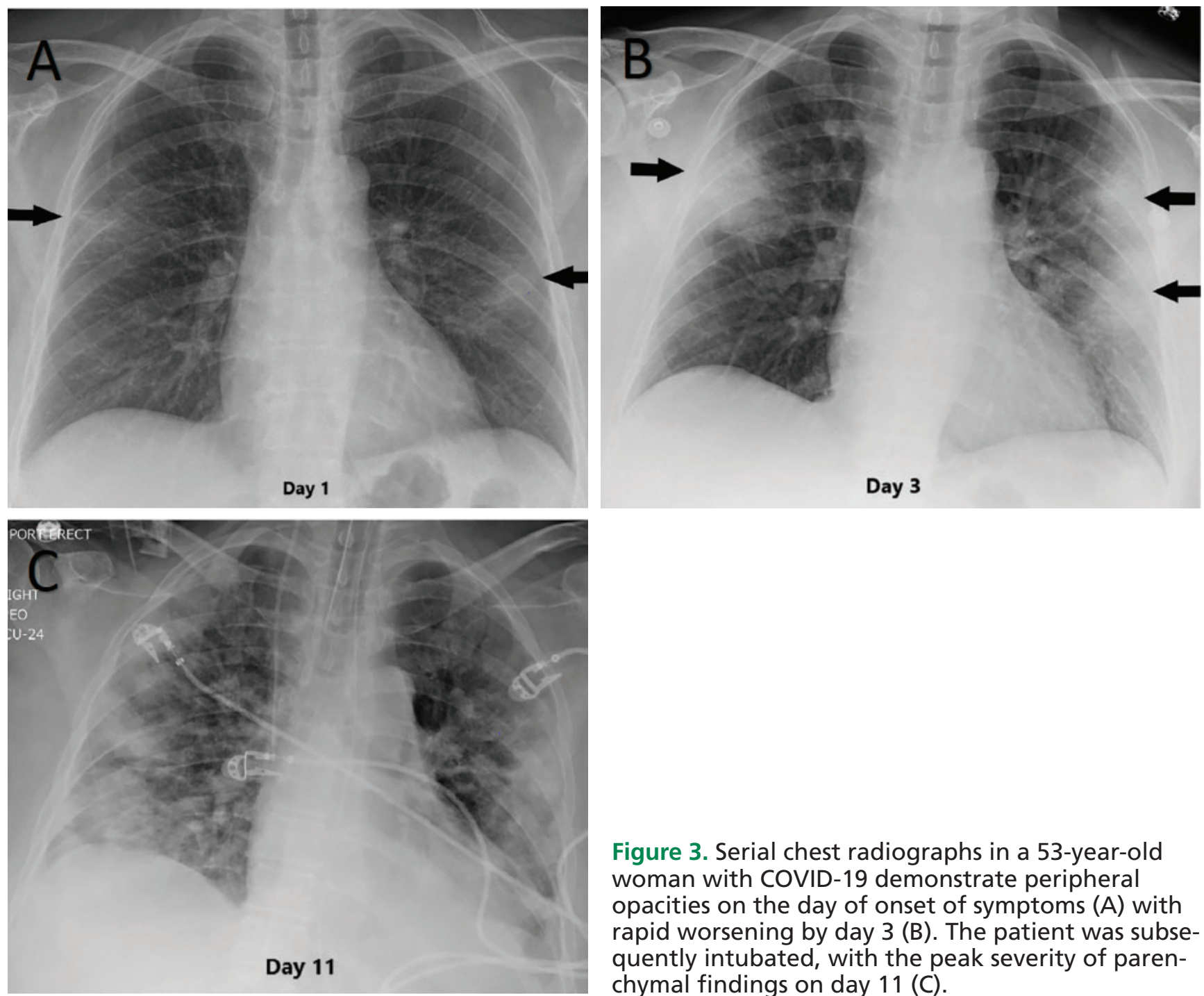

Figure 3. Serial chest radiographs in a 53-year-old woman with COVID-19 demonstrate peripheral opacities on the day of onset of symptoms (A) with rapid worsening by day 3 (B). The patient was subsequently intubated, with the peak severity of parenchymal findings on day 11 (C).

of pneumonia caused by other coronaviruses such as SARS and MERS, as well as other bacterial and viral infections, most notably influenza pneumonia. Moreover, noninfectious etiologies such as drug toxicities and connective tissue disease may produce similar imaging findings. Hence, integration of the clinical presentation, exposure history, prevalence of COVID-19 in the community, and personal risk factors are of paramount importance in suspecting and making the diagnosis.

The most characteristic CT findings of COVID-19 pneumonia are ground-glass opacities with or without consolidation and superimposed interlobular septal thickening (crazypaving appearance). A reverse halo (central ground-glass opacities with an interrupted peripheral rim of consolidation) has also been described, especially in the later stages of the disease. These lung opacities are frequently bilateral, multilobar, posterior, peripheral, and basilar in distribution and often rounded in morphology (Figure 5). . $^{3-9}$

Over time, the ground-glass opacities may worsen, with progressive consolidation and more lobes involved. The severity of the CT findings peaks 10 to 12 days after the onset of symptoms. ${ }^{5}$ A subset of hospitalized patients with COVID-19 (16.1\% in one series) develop extensive lung disease with acute respiratory distress syndrome (ARDS). ${ }^{10}$ Table 1 summarizes the CT findings in the different 

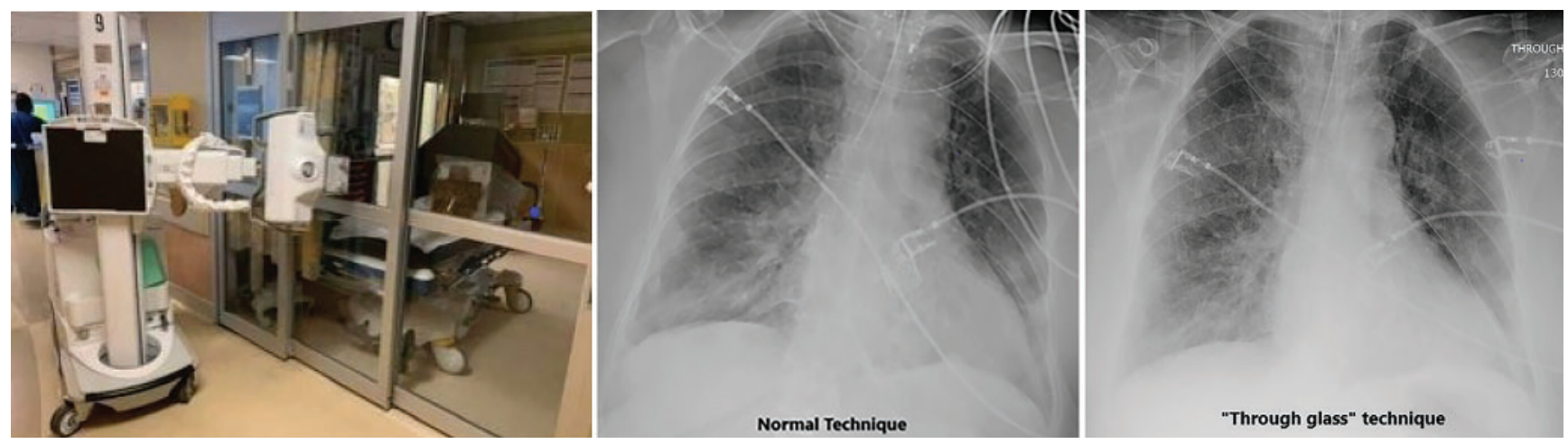

Figure 4. (Left) Setup for obtaining chest radiographs through the glass wall of the room of a patient with suspected or confirmed COVID-19. (Middle) A radiograph obtained in the conventional manner. (Right) A radiograph obtained through the glass.

stages of COVID-19, including recovery (Figure 6). ${ }^{5,11-14}$

Gattinoni et $\mathrm{al}^{15}$ describe 2 different phenotypes in patients with COVID-19: type L, characterized by low elastance, low ventilation-to-perfusion ratio, low lung weight, low recruitability, and CT findings of subpleural opacities; and type $\mathrm{H}$, characterized by high elastance, high ventilation-to-perfusion ratio, high lung weight, high recruitability, and CT findings of ARDS. Understanding the differ-

Chest

radiography

is considered

an appropriate

initial

diagnostic

imaging test

for most

patients ent pathophysiology is crucial for appropriate patient management.

Thrombotic complications (pulmonary embolism, deep vein thrombosis, ischemic cerebrovascular accident, and myocardial infarction) have emerged as important sequelae that contribute to morbidity and mortality in COVID-19 patients. There is an increased incidence of pulmonary embolism in patients with confirmed COVID-19 disease (Figure 7), with rates ranging from $23 \%$ to $37 \%$ in the recent literature. ${ }^{16-18}$ One of the studies ${ }^{19}$ showed that body mass index greater than $30 \mathrm{~kg} / \mathrm{m}^{2}$, increase in D-dimer greater than $6 \mu \mathrm{g} / \mathrm{mL}$, and history of hypertension or prior pulmonary embolism were associated with increased risk of pulmonary embolism in COVID-19.

Twenty percent of COVID-19 patients may have coexistent infections complicating the characterization of imaging observations. ${ }^{20}$ Hence, the radiologist has to determine whether or not these findings are part of the same process or are unrelated. ${ }^{20}$

As with most other viral pneumonias, features not typically seen in COVID-19 include pleural effusion, lymphadenopathy, cavitation, and small discreet nodules (including centrilobular or tree-in-bud opacities)..$^{21,22}$

\section{THE RADIOLOGICAL SOCIETY OF NORTH AMERICA CONSENSUS STATEMENT}

The Radiological Society of North America (RSNA) published a consensus statement in March 2020 on standardized reporting of CT findings related to COVID-19. It had several goals, including to reduce uncertainty and variability in reporting findings potentially attributable to COVID-19, and to enhance the referring providers' understanding of common radiographic findings. ${ }^{23}$

Typical features, according to the RSNA report, are lower-lobe-predominant, peripheral-predominant, multiple, bilateral foci of rounded ground-glass opacities with or without crazy paving, peripheral consolidation, and a reverse halo perilobular pattern (seen later in the disease).

Indeterminate features are absence of typical features and the presence of multifocal, diffuse, perihilar, or unilateral groundglass opacities with or without consolidation, lacking a specific distribution and that are nonrounded or nonperipheral. Another: few very small ground-glass opacities with a nonrounded and nonperipheral distribution.

Atypical features are absence of typical or indeterminate features and the presence of isolated lobar or segmental consolidation without ground-glass opacities, discrete small nodules (centrilobular, tree-in-bud), lung cavitation, or smooth interlobular septal thicken- 


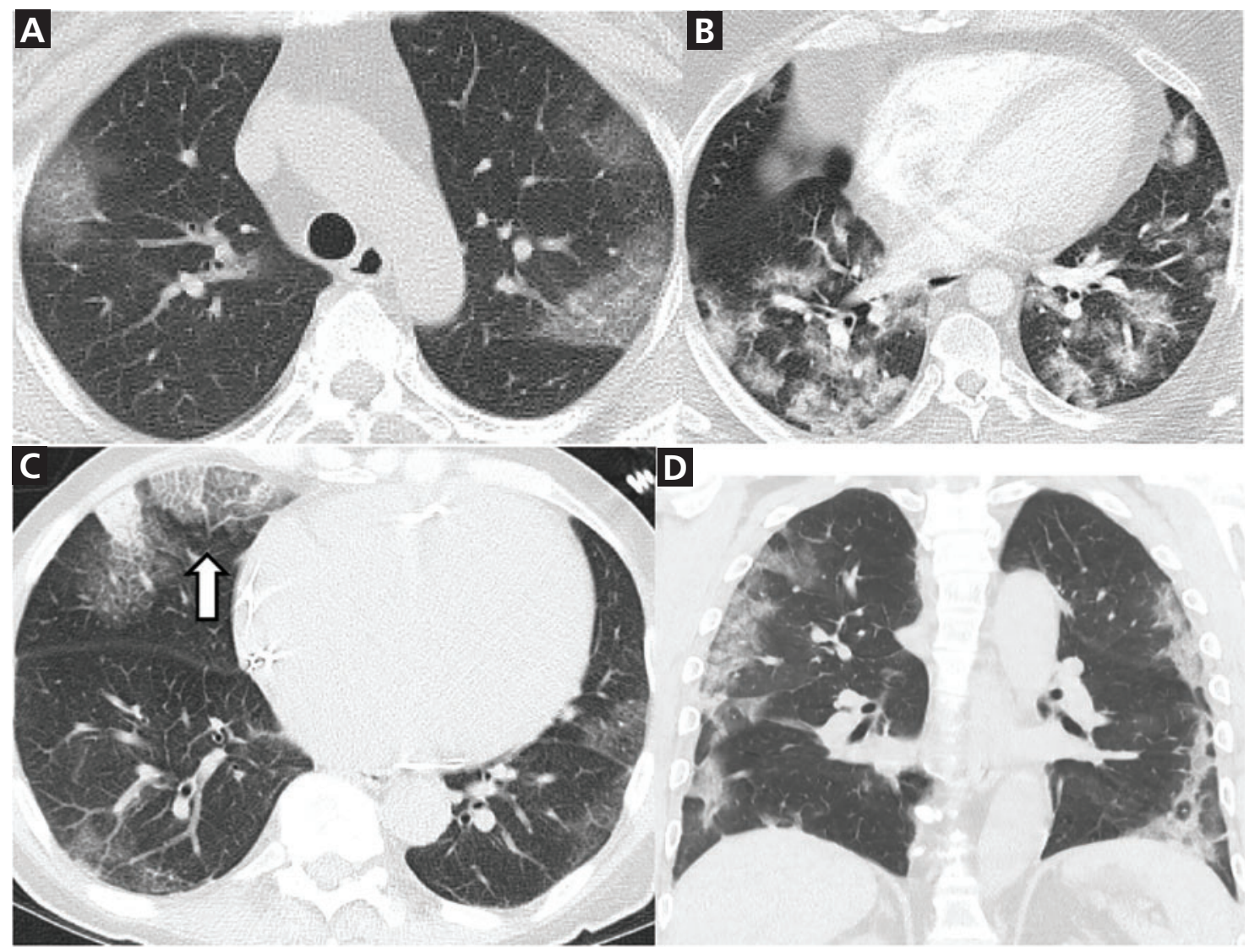

Figure 5. Typical computed tomographic features of COVID-19. Unenhanced axial images of the lungs of 4 different patients with COVID-19 demonstrate bilateral, multifocal, peripheral ground-glass opacities, and consolidation, most with rounded morphology. "Crazy paving" (ground-glass opacities with superimposed interlobular septal thickening and intralobular lines) is seen in (C) (white arrow).

ing with pleural effusion.

Caveats. The statement advises including cautionary language in radiographic reports. If typical features of COVID-19 are present, for example, the statement advises mentioning that other processes can also cause a similar imaging pattern. If indeterminate features are seen, the statement points out that these features are nonspecific. Even if no findings are present to indicate pneumonia, it notes that CT may be negative in the early stages of COVID-19.

\section{ROLE OF CT IN COVID-19}

The sensitivity of chest CT in detecting COVID-19 pneumonia has been reported to be $97 \%$, the specificity $25 \%$ to $56 \%$, and the accuracy $68 \%$ to $72 \%,{ }^{9,21,24,25}$ CT findings may be negative in $20 \%$ to $25 \%$ of patients early in the course of the disease. Hence, a normal chest CT scan cannot exclude the diagnosis of COVID-19, especially early after the onset of symptoms. ${ }^{25}$ CT has been reported to become abnormal in more than $95 \%$ of cases after 5 to 6 days of infection.

The available literature suggests that CT imaging may be helpful in early detection of pneumonia in patients in whom COVID-19 is strongly suspected but who have an initial false-negative RT-PCR screening test. ${ }^{21,26,27}$

\section{CURRENT RECOMMENDATIONS}

Most radiology professional organizations and societies as well as the US Centers for Disease Control and Prevention currently recommend against performing routine screening CT to diagnose or exclude COVID-19.23,28
Chest radiography has a high false-negative rate, especially in the early stage of infection 


\section{TABLE 1}

Stages of COVID-19 on chest CT

Early stage (0-2 days)

Approximately $50 \%$ of patients have negative chest CT

The remaining have ground-glass opacities (44\%) and consolidation $(17 \%)$, more often unilateral

The less pulmonary consolidation identified on $\mathrm{CT}_{\text {, }}$ the greater the probability of initial negative reverse transcriptase polymerase chain reaction results ${ }^{11}$

Intermediate stage (3-5 days)

$9 \%$ of patients have negative chest CT

$88 \%$ have ground-glass opacities with or without crazy paving (a sign of progression or peak stage), and $55 \%$ have consolidation (bilateral in $76 \%$, peripheral in distribution in $64 \%$ with rounded morphology $)^{12}$

Late phase (6-12 days)

Most patients have positive $\mathrm{CT}$ findings

Progressive consolidation, evolving linear consolidation, and organizing pneumonia

Reverse-halo appearance (a sign of healing or evolving lesion) $)^{12}$

Ground-glass opacities in $88 \%$ with or without crazy paving

\section{Severe phase}

Massive pulmonary consolidation and "white lungs"

\section{Recovery phase}

Parenchymal abnormalities resolve with residual linear opacities (Figure 6)

Based on information from references 5 and 11-14.

CT is commonly indicated for hospitalized, symptomatic patients with worsening respiratory status or in patients with moderate to severe features of COVID-19 regardless of the COVID-19 test results. ${ }^{23,28}$ The societies also suggest that hospitals consider deploying portable radiography units in ambulatory care facilities for use when chest radiographs are considered medically necessary. The surfaces of these machines can be easily cleaned, avoiding the need to bring patients into radiography rooms.

\section{FLEISCHNER SOCIETY STATEMENT}

In a multinational consensus statement, the Fleischner Society states that in a resourceconstrained environment (in which personal protective equipment or COVID-19 testing may not be available), imaging is indicated for medical triage of patients with suspected COVID-19 who present with moderate to severe clinical features and a high pretest probability of the disease..$^{28}$

The report further states that imaging is not routinely indicated as a screening test for COVID-19 in patients without symptoms, nor for patients with mild features of COVID-19 unless they are at risk for disease progression. Imaging is indicated for patients with moderate to severe features of COVID-19 regardless of the COVID-19 test results, and for patients with COVID-19 and evidence of worsening respiratory status. In a resource-constrained environment where access to CT is limited, chest radiography may be preferred for patients with COVID-19 unless features of respiratory worsening warrant the use of CT.

Daily chest radiographs are not indicated in stable intubated patients with COVID-19. CT is indicated in patients with functional impairment or hypoxemia after recovery from COVID-19. And COVID-19 testing is indicated in patients incidentally found to have findings suggestive of COVID-19 on a CT scan. ${ }^{28}$

All patients undergoing imaging should be masked. ${ }^{29}$ Appropriate infection-control procedures should be followed before scanning subsequent patients. ${ }^{23}$ 


\section{YADAV AND COLLEAGUES}
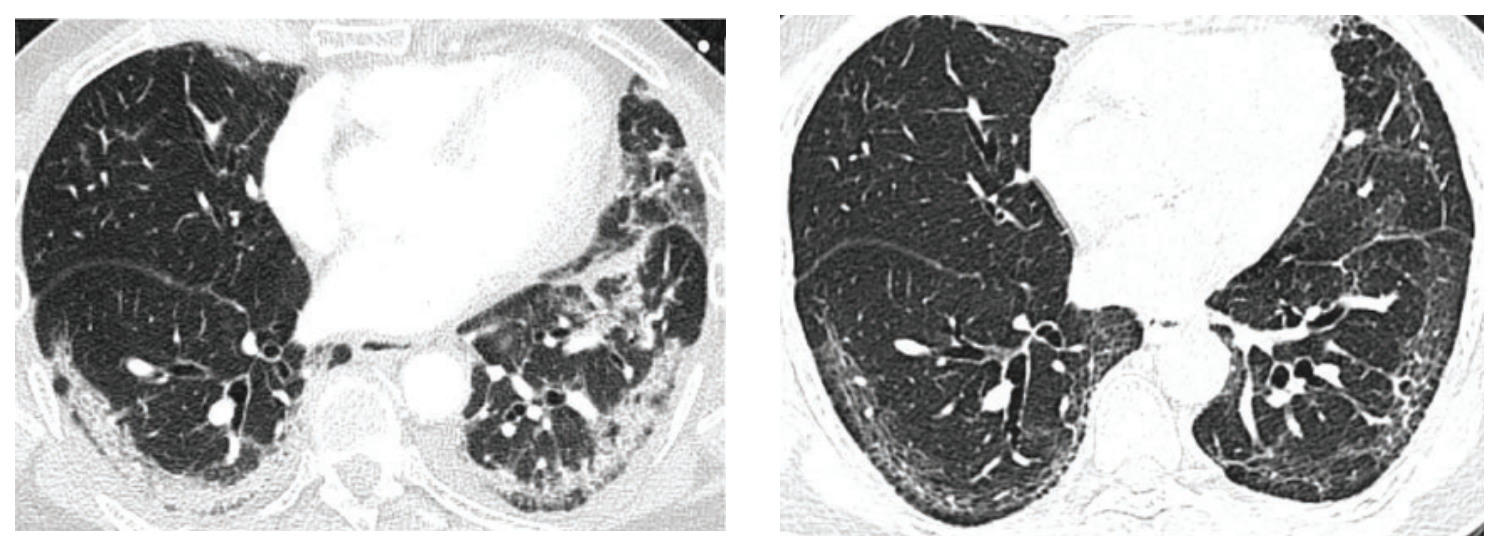

Figure 6. CT scans in a 73-year-old man with COVID-19 demonstrate resolution of subpleural consolidation with residual reticular and fibrotic changes.
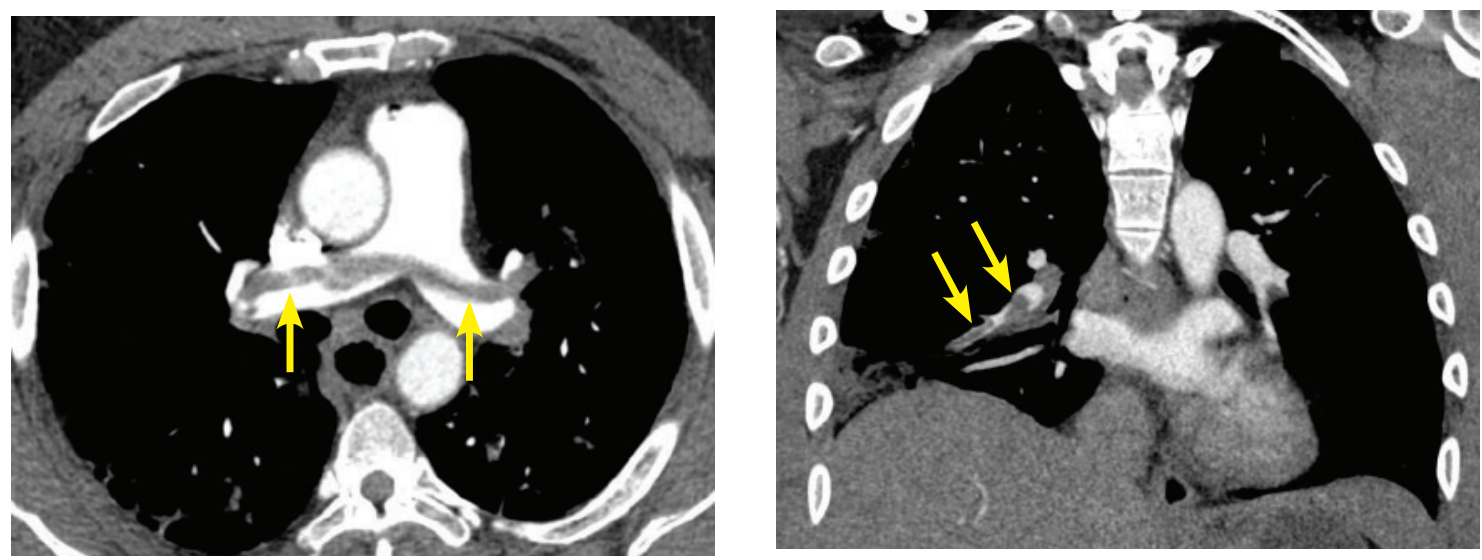

Figure 7. CT images of 2 different patients with COVID-19 demonstrating pulmonary embolism.

\section{REFERENCES}

1. Wong HYF, Lam HYS, Fong AH, et al. Frequency and distribution of chest radiographic findings in COVID-19 positive patients. Radiology 2019 Mar 27;201160. doi:10.1148/radiol.2020201160

2. Mozdy M. Safer, PPE-conserving x-rays for patients at University Hospital. U Health University of Utah School of Medicine. April8, 2020. Accessed July 14, 2020. https://medicine.utah.edu/radiology/ news/2020/04/x-ray-through-glass.php

3. Chung M, Bernheim A, Mei X, et al. CT imaging features of 2019 novel coronavirus (2019-nCoV). Radiology 2020; 295(1):202-207. doi:10.1148/radiol.2020200230

4. Kong W, Agarwal PP. Chest imaging appearance of COVID-19 infection. Radiology Cardiothoracic Imaging 2020 February 13. doi:10.1148/ryct.2020200028

5. Bernheim A, Mei X, Huang M, et al. Chest CT findings in coronavirus disease-19 (COVID-19): relationship to duration of infection. Radiology 2020; 295(3):200463. doi:10.1148/radiol.2020200463

6. Pan $F, Y e T$, Sun $P$, et al. Time course of lung changes at chest $C T$ during recovery from 2019 novel coronavirus (COVID-19) pneumonia. Radiology 2020; 295(3):715-721. doi:10.1148/radiol.2020200370

7. Bai HX, Hsieh B, Xiong Z, et al. Performance of radiologists in differentiating COVID-19 from viral pneumonia on chest CT. Radiology 2020 Mar 10;200823. doi:10.1148/radiol.2020200823

8. Salehi S, Abedi A, Balakrishnan S, Gholamrezanezhad A. Coronavirus disease 2019 (COVID-19): a systematic review of imaging findings in 919 patients. AJR Am J Roentgenol 2020;215(1):87-93.
doi:10.2214/AJR.20.23034

9. Caruso D, Zerunian M, Polici M, et al. Chest CT features of COVID-19 in Rome, Italy. Radiology 2020 Apr 3;201237. doi:10.1148/radiol.2020201237

10. Wang D, Hu B, Hu C, et al. Clinical characteristics of 138 hospitalized patients with 2019 novel coronavirus-infected pneumonia in Wuhan, China. JAMA 2020; 323(11):1061-1069. doi: 10.1001/jama.2020.1585

11. Chen $D$, Jiang $X$, Hong $Y$, et al. Can chest $C T$ features distinguish patients with negative from those with positive initial RT-PCR results for coronavirus disease (COVID-19)? AJR Am J Roentgenol 2020 May 5;1-5. doi:10.2214/AJR.20.23012

12. Bai HX, Wang R, Xiong Z, et al. Al Augmentation of radiologist performance in distinguishing COVID-19 from pneumonia of other etiology on chest CT. Radiology 2020 Apr 27;201491. doi:10.1148/radiol.2020201491

13. Zhou S, Wang Y, Zhu T, Xia L. CT features of coronavirus disease 2019 (COVID-19) pneumonia in 62 patients in Wuhan, China. AJR Am J Roentgenol 2020; 214(6):1287-1294. doi:10.2214/AJR.20.22975

14. Hani C, Trieu NH, Saab I, et al. COVID-19 pneumonia: a review of typical CT findings and differential diagnosis. Diagn Interv Imaging 2020; 101(5):263-268. doi:10.1016/j.diii.2020.03.014

15. Gattinoni L, Chiumello D, Caironi P, et al. COVID-19 pneumonia: different respiratory treatment for different phenotypes? Intensive Care Med 2020; 46(6):1099-1102. doi:10.1007/s00134-020-06033-2

16. Grillet F, Behr J, Calame P, Aubry S, Delabrousse E. Acute pulmonary 
embolism associated with COVID-19 pneumonia detected by pulmonary CT angiography. Radiology 2020; Apr 23;201544. doi:10.1148/radiol.2020201544

17. Cheng $\mathbf{Y}$, Luo R, Wang $K$, et al. Kidney disease is associated with in-hospital death of patients with COVID-19. Kidney Int 2020; 97(5)829-838. doi:10.1016/j.kint.2020.03.005

18. Kaminetzky M, Moore W, Fansiwala K, James S, et al. Pulmonary embolism on CTPA in COVID-19 patients. Radiology: Cardiothoracic Imaging 2020 Jul 2. doi:10.1148/ryct.2020200308

19. Poyiadji N, Cormier P, Patel PY, et al. Acute pulmonary embolism and COVID-19. Radiology 2020; May 14;201955. doi:10.1148/radiol.2020201955

20. Shah N. Higher co-infection rates in COVID19. Medium. Accessed July 16, 2020. https://medium.com/@nigam/higher-co-infectionrates-in-covid19-b24965088333

21. Ai T, Yang Z, Hou H, et al. Correlation of chest CT and RT-PCR testing in coronavirus disease 2019 (COVID-19) in China: a report of 1014 cases. Radiology 2020 Feb 26;200642. doi:10.1148/radiol.2020200642

22. Ng M, Lee $\mathbf{E}$, Yang J, et al. Imaging profile of the COVID-19 infection: radiologic findings and literature review. Radiology Cardiothoracic Imaging 2020 Feb 13. doi:10.1148/ryct.2020200034

23. Simpson S, Kay F, Abbara S, et al. Radiological Society of North America expert consensus statement on reporting chest CT findings related to COVID-19. Endorsed by the Society of Thoracic Radiology, the American College of Radiology, and RSNA. Radiology 2020; 35(4):219-227. doi:10.1097/RTI.0000000000000524
24. Cheng Z, Lu Y, Cao Q, et al. Clinical features and chest CT manifestations of coronavirus disease 2019 (COVID-19) in a single-center study in Shanghai, China. AJR Am J Roentgenol 2020; 215(1)121-126. doi:10.2214/AJR.20.22959.

25. Fang Y, Zhang H, Xie J, et al. Sensitivity of chest CT for COVID-19: comparison to RT-PCR. Radiology 2020 Feb 19;200432. doi:10.1148/radiol.2020200432

26. Huang $P$, Liu $T$, Huang $L$, et al. Use of chest $C T$ in combination with negative RT-PCR assay for the 2019 novel coronavirus but high clinical suspicion. Radiology 2020; 295(1):22-23. doi:10.1148/radiol.2020200330

27. Xie X, Zhong Z, Zhao W, Zheng C, Wang F, Liu J. Chest CT for typical 2019-nCoV pneumonia: relationship to negative RT-PCR testing. Radiology 2020 Feb 12;200343. doi:10.1148/radiol.2020200343

28. Rubin GD, Ryerson CJ, Haramati LB, et al. The role of chest imaging in patient management during the COVID-19 pandemic: a multinational consensus statement from the Fleischner Society. Radiology 2020; 296(1):172-180. doi:10.1148/radiol.2020201365

29. Kooraki S, Hosseiny M, Myers L, Gholamrezanezhad A. Coronavirus (COVID-19) outbreak: what the department of radiology should know. J Am Coll Radiol 2020; 17(4):447-451. doi:10.1016/j.jacr.2020.02.008

Address: Ruchi Yadav, MD, Diagnostic Radiology, L10, Cleveland Clinic 9500 Euclid Avenue, Cleveland, OH 44195; yadavr@ccf.org 